\title{
Enhancing outcomes of mandibular reconstruction by introducing three-dimensional printing
}

\author{
Mihai Liviu Ciofu' ${ }^{1}$ Andrei Rosu², Cristian Ilie Drochioi', Andrada Doscas', \\ Otilia Boisteanu', Victor-Vlad Costan ${ }^{1}$ \\ ${ }^{1}$ Oral and Maxillofacial Surgery Department, \\ "Grigore T. Popa" University of Medicine and Pharmacy, Iasi, Romania \\ ${ }^{2}$ Otolaryngology Department, "Grigore T. Popa" University of Medicine and Pharmacy, lasi, Romania
}

\begin{abstract}
Aim of the study. This study focuses on the advantages of including three-dimensional printing in the preoperative planning of mandibular reconstruction procedures following the removal of malignant tumors.

Material and methods. We performed a retrospective study including patients with mandibular defects due to oncologic resections that underwent reconstruction procedures using stereolithic models in the preoperative stage. The general information, diagnosis, the technique of obtaining the stereolithic models and their use, as well as the type of surgical procedure and the outcomes were all documented.

Results. In all seven included cases mandibular reconstruction was performed using a titanium reconstruction plate that was preoperatively shaped according to the 3D printed stereolithic models. The pre-bent plates adapted well to the defect intraoperatively. A decreased overall surgical time was noted. The aesthetic and functional reconstructive outcomes were favorable.

Conclusions. The use of three-dimensional printing has proven advantageous in our experience for increasing the predictability and quality of the mandibular reconstructions.
\end{abstract}

Keywords: mandible, reconstruction, defect, 3D printing, stereolithic model, preoperative planning

\section{INTRODUCTION}

The integrity and harmony of the facial components are important features since they contribute to physiological functions' exertion as well as interpersonal recognition and relations. Any facial defect results in functional and aesthetic deficiencies that are easily observed.

This article focuses on enhancing mandibular reconstruction after tumoral resection by using threedimensional (3D) printing techniques in the preoperative stage. The resection of the mandible is influenced by decisions and indications that are determined in context of many clinical variables recorded through routine and comprehensive physical and imaging examinations (1). After a segmental mandibular bone resection, rehabilitation of the mandibular contour, the reconstruction of the occlusion with the correct position of the temporal-mandibular joint condyles in the glenoid fossae are crucial to provide the patient adequate masticatory function and an aesthetically satisfying result. Therefore, mandibular reconstruction remains among the most challenging endeavors faced by reconstructive surgery (2).

Given the increased demands on restoring facial harmony and normal functionality, with continuous development of the imaging and therapeutic methods used for this purpose, it is necessary to permanently re-evaluate and reorganize the multiple existing resources, in order to optimize the time needed to solve the case, with optimal results.

With the recent advancements in computer aided medicine, numerous studies focus on evaluating the use of 3D printers and stereolithographic models in various areas of the medical field (3). The purpose of this article is to present our experience regarding the use of stereolithic models for simplifying the reconstruction of mandibular defects. 


\section{MATERIAL AND METHODS}

We reviewed the medical charts of patients with mandibular defects due to malignant tumor resection that underwent mandibular reconstruction procedures in which three-dimensional printing was used in the preoperative stage. The general patient information, the diagnosis, the preoperative planning, the type of surgical procedure and the outcomes were all documented.

\section{RESULTS}

\section{Patients}

A total of 7 patients were included in the study, of which 6 men and 1 woman, aged between 39 and 63 years old. Three patients were diagnosed with gingivoalveolar carcinomas, while 4 patients had floor of the mouth carcinomas extended to the mandible. All patients were evaluated for the amount of tumor extension and bone invasion using a preoperative CT (computed tomography) scan.

\section{Technique}

For obtaining the stereolithic models, a virtual model was first constructed using the CT information. Initially, case evaluation and planning were performed by analyzing the $3 \mathrm{D}$ reconstruction of the CT images and establishing the extent of bone resection necessary. Mirroring of the unaffected side over the defect side was performed in 2 cases in order to achieve an ideal virtual model as a representation of the final reconstruction shape. In the other 5 cases, the actual unmodified $3 \mathrm{D}$ reconstruction of the patient's mandible was used for printing the model, since the mandibular shape remained unaltered by tumor infiltration. The virtual information was then sent to the 3D printer for transformation into the physical object the stereolithic model. This printed model was then used preoperatively for shaping the reconstruction plate. In 4 cases, guides for positioning of the reconstruction plate during surgery were also modelled preoperatively using the printed model.

The surgical procedure consisted of a segmental mandibular resection in all cases. In all patients the resection spared the mandibular condyle. The continuity of the mandible was restored using a titanium reconstruction plate that was modelled the day before surgery, using the patient specific 3D printed stereolithic model.

\section{Outcomes}

The preshaped reconstruction plates were adequately modelled in all cases and did not require any intraoperative adaptation. The guides were useful in positioning the reconstruction plates in the planned position, assuring the predicted results. The overall operative time was shortened due to the absence of the intraoperative reconstruction plate bending stage and predrilling of the fixation screw location before bone resection. The confidence of the surgical team was increased due to the preoperative preview of the patient's anatomy.

An accurate restoration of the mandibular shape was achieved in all the included cases, resulting in good aesthetic and functional results.

The favorable outcomes are presented by images of a clinical case (Fig. 1-7), representing a patient with a gingivoalveolar carcinoma of the right hemimandible extended to the anterior arch that was surgically removed by segmental mandibular resection (from 34 to the right angle supra-Spix "L" osteotomy) with concurrent bilateral neck dissection and primary reconstruction of the mandible continuity using a titanium reconstruction plate and a pectoralis major musculocutaneous pedicled flap. The titanium reconstruction plate was modelled prior to surgery using a patient specific 3D printed stereolithic model.

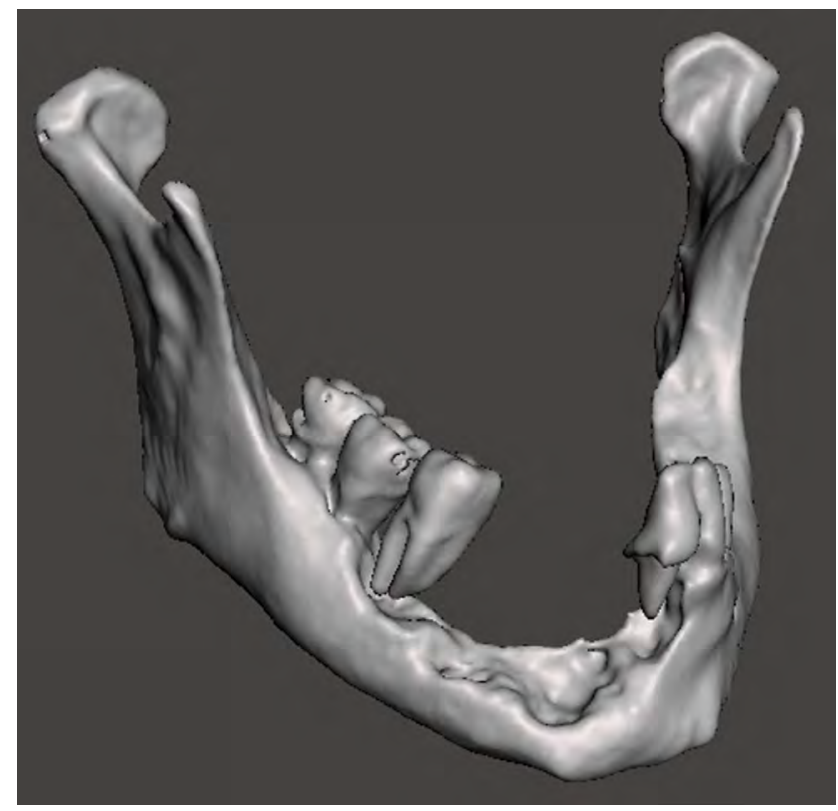

FIGURE 1. Oblique view of the $3 D$ virtual model of the patient's mandible used during preoperative virtual planning 


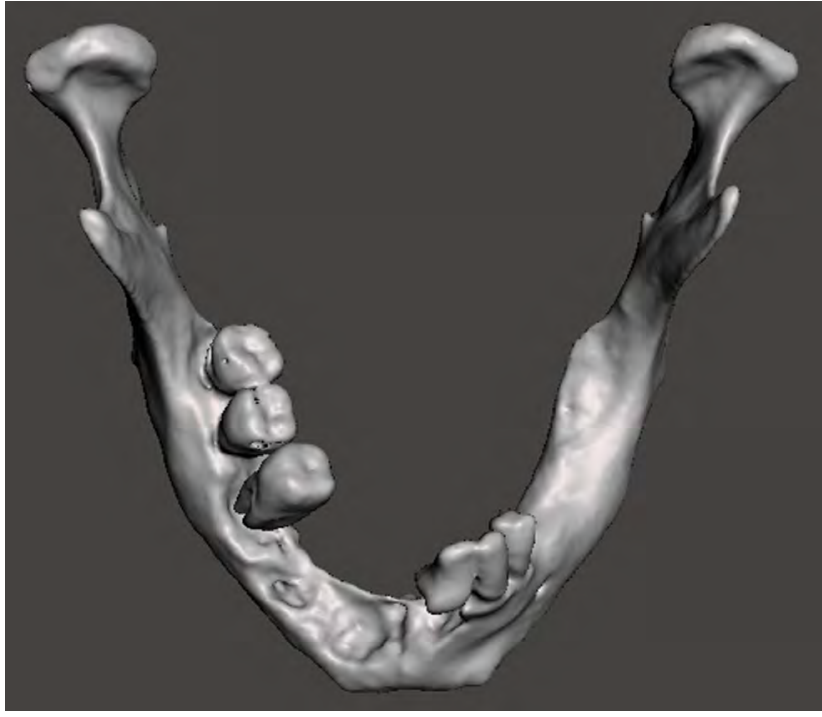

FIGURE 2. Superior view of the $3 D$ virtual model

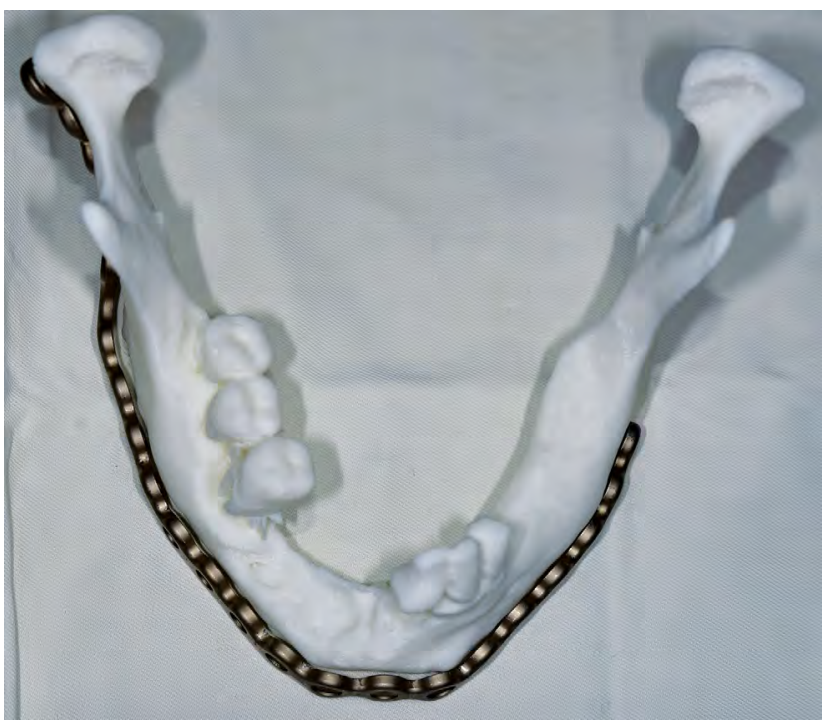

FIGURE 3. Superior view of the preoperatively modelled reconstruction plate on the $3 D$ printed stereolithic model

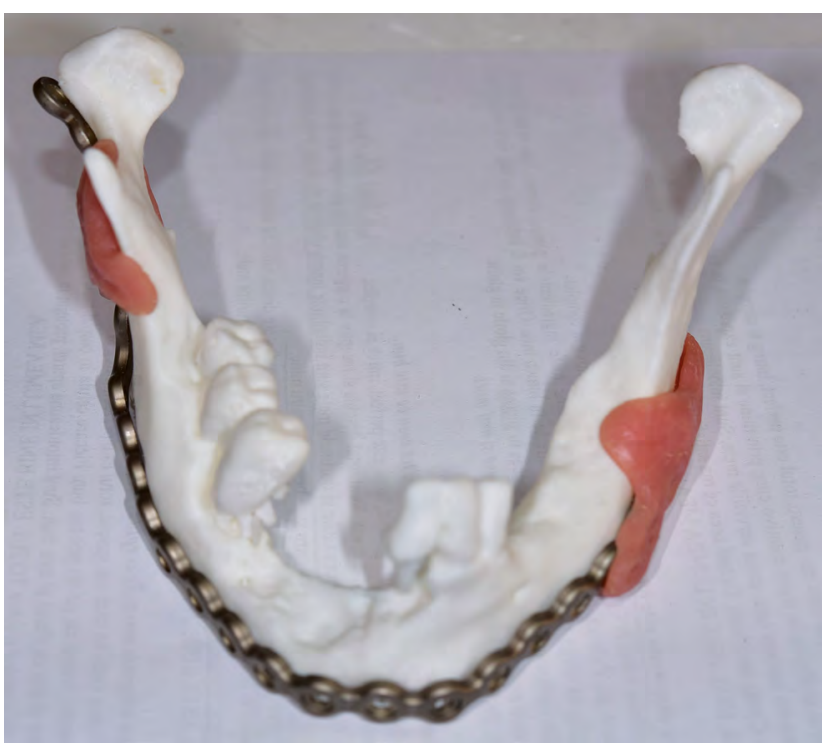

FIGURE 4. Superior view of the preoperatively modelled reconstruction plate and surgical guides on the $3 D$ printed stereolithic model

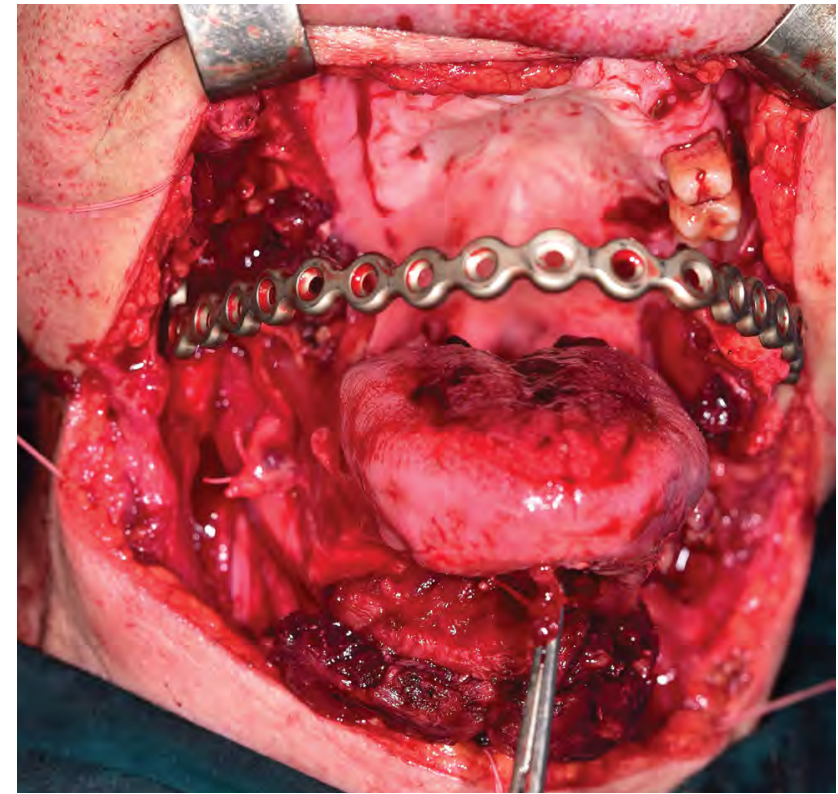

FIGURE 5. Intraoperative view of the pre-bent titanium reconstruction plate in place after fixation

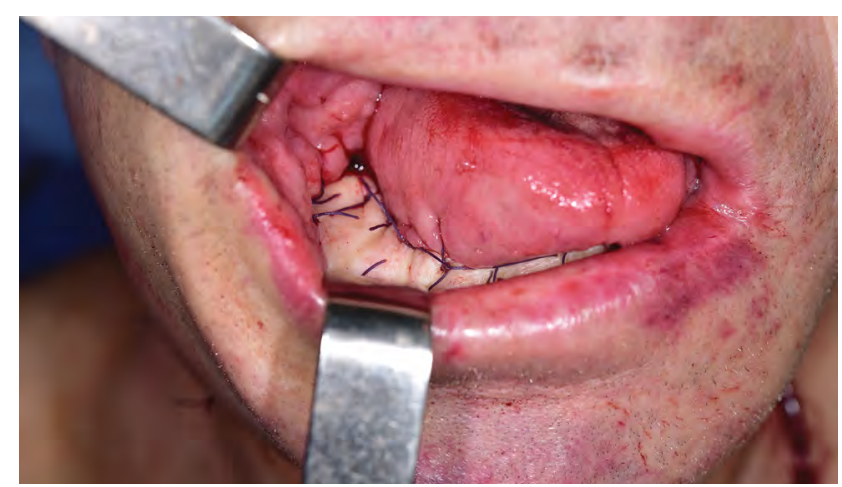

FIGURE 6. Postoperative intraoral view of the cutaneous paddle of the pectoralis major flap covering the reconstruction plate.

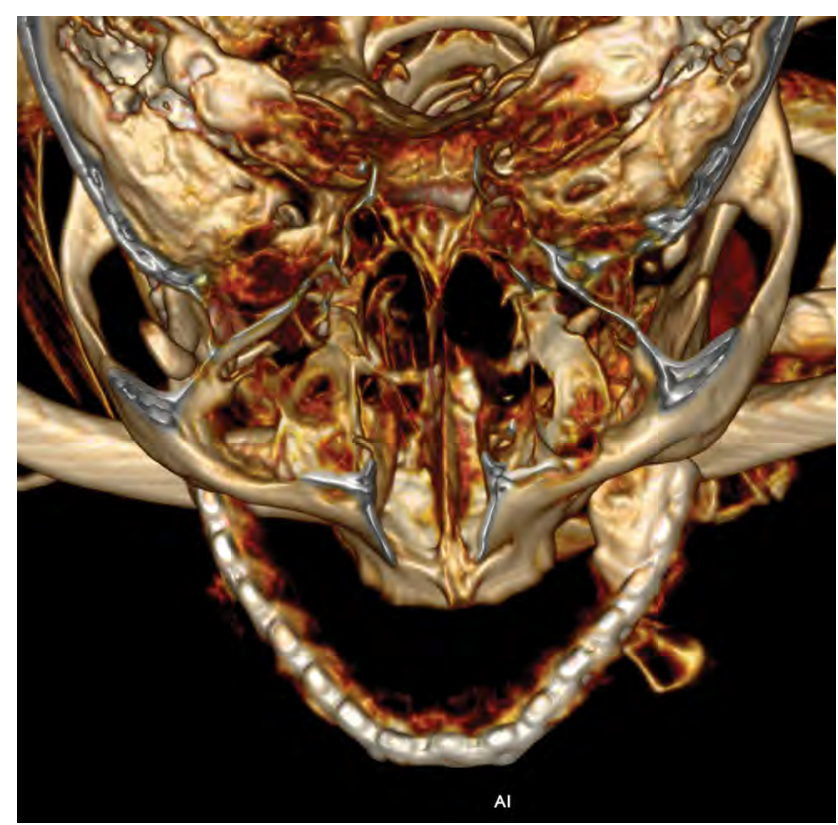

FIGURE 7. Postoperative 3D CT reconstruction (superior view) showing the accurately modelled mandibular titanium plate 


\section{DISCUSSIONS}

Patient specific reconstructions have become increasingly popular in cranio-maxillofacial surgery. The recently available technical equipment and software allow the relatively easy transformation of the images obtained by a CT scan into 3D models. In order to accomplish the transformation, it is mandatory that the patient is subjected to a CT or CBCT (Cone Beam Computed Tomography) examination. The acquired Digital Imaging and Communications in Medicine (DICOM) data is transferred into the computer software thus obtaining a true virtual 3D model of the patient's mandible. Subsequently, the operating steps can be simulated on the computer (4-6).

The stereolitography (STL) data file format, introduced in 1987 by 3D Systems as a data transmission format for its first commercially available 3D printer, describes the surface of an object as an unstructured appearing triangular representation $(3,4,6)$. The advancement in computer-assisted design software accounts the utility of two-dimensional (2D) data from a computed tomography (CT) scan to generate a three-dimensional virtual representation of a given body part that should be comprehensible. Rendering the $2 \mathrm{D}$ slice images from a CT scan into a $3 \mathrm{D} C \mathrm{CT}$ reconstruction in DICOM format, then translating that file information with a suitable software into a STL-format, results in a 3D construct. Furthermore, the thinner the CT slice thickness and as more slices the CT set enumerates, the more point coordinates are presented for a detailed 3D model (4). By means of further information taken from the modality worklist of a DICOM file, the converted STL construct visualization can be additionally refined and through software manipulation, the 3D model of the patient mandible is virtually cut, allowing the surgeon to conduct preoperative training $(3,4)$. In context of mandibular reconstruction, it is usually a true-to-size replica of the bone donor graft area or/ and of the bone graft recipient side and a template of the area including the defect can be modelled. Furthermore, with the help of 3D printing technology, a stereolithographic model of this template can be obtained, sterilized and used during the operation.

The approach to mandibular reconstruction is different among surgeons, but most methods rely on reconstructing the continuity of the mandible using either a titanium reconstruction plate, or an association of a bone flap or graft and fixation material. The golden standard for reconstructing large mandibular defects is the free vascularized flap $(7,8)$. This allows for the reconstruction of a large segment of the mandible as well as reducing the time needed for osseointegration of the donor flap and a minimal risk of bone resorption, but comes with the disadvantage of morbidity of the donor site, thus wasting as little as possible tissue should be considered.

The use of 3D printing techniques can offer multiple advantages to mandibular reconstruction procedures $(5,9,10)$. They allow the preoperative modelling of the material used for reconstruction or fixation of the graft or bone flap used. Threedimensional printing can even be used for estimating the amount and shape of the bone to be used for the reconstruction $(10,11)$. Classical reconstruction procedures involve a long operation time, increasing the risk of a prolonged period of tissue ischemia and not achieving a sufficient bone-to-bone contact, incorrect maxillo-mandibular and occlusal relationships and can ultimately lead to a higher rate of complications, poor aesthetic and poor functional results $(12,13)$. The obtained 3D printed model of the defect offers the surgeon not only the information about the osseous material that is needed to be harvested, but also about the shape and size of the plates needed for securing the osseous flap.

In the case of oncologic patients, many authors advocate for performing bone reconstruction at a distance from tumor resection, in order to ensure the absence of recurrence. The primary reconstruction is thus often performed using only a thick titanium reconstruction plate that is well covered by soft tissues $(1,11)$. Traditionally, the shaping of the reconstruction plate is accomplished intraoperative, with the original mandible as a template. Pilot drill holes are also usually performed in order to help position the modelled plate after the segmental bone resection. Performing these steps is challenging during surgery due to technical aspects related to the wear of gloves, wet environment, the thickness of the plate and the sharp instruments used (5). Some authors mention that the procedure may take up to 60 minutes from overall surgery time due to the complex 3-dimensional shape of the mandible (14). 
The 3D printed stereolithic models allow for a decrease of the operation time as well as improving the aesthetics and functionality due to the preoperative planning and plate bending (15). Some authors reported an estimated time gain of approximately 30 minutes in case of titanium plate reconstruction and 1,5 to 2 hours for fibula free flap mandibular reconstruction, with flap ischemia time also reduced (16). Such results are similar to the ones in our study, since surgery time was overall decreased by the pre-bending of the plates using the stereolithic model in a preoperative stage. An additional possible cause for the reduced surgery time is the increased confidence of the surgical team that had better comprehension of the case's anatomy due to physical involvement in the preoperative planning and plate bending using the patient specific model. Other studies also mention that the preoperative planning made possible by the manipulation of the 3D model, physical or through software, increases the confidence of the surgical team due to the pre surgery preview of the patient's anatomy improving the predictability of the clinical outcome when compared with similar treatments without its use $(9,17)$.

\section{REFERENCES}

1. Batstone MD. Reconstruction of major defects of the jaws. Aust Dent J. 2018;63 Suppl 1:S108-S113.

2. Zhao L, Patel PK, Cohen M. Application of virtual surgical planning with computer assisted design and manufacturing technology to cranio-maxillofacial surgery. Arch Plast Surg. 2012;39(4):309-16.

3. Louvrier A, Marty P, Barrabé A, Euvrard E, Chatelain B, Weber E, Meyer $C$. How useful is $3 \mathrm{D}$ printing in maxillofacial surgery? J Stomatol Oral Maxillofac Surg. 2017;118(4):206-212.

4. Ciocca L, Mazzoni S, Fantini M, Persiani F, Marchetti C, Scotti R. CAD/CAM guided secondary mandibular reconstruction of a discontinuity defect after ablative cancer surgery. J Craniomaxillofac Surg. 2012;40(8):e511-5.

5. Azuma M, Yanagawa T, Ishibashi-Kanno N, Uchida F, Ito T, Yamagata K, Hasegawa S, Sasaki K, Adachi K, Tabuchi K, Sekido M, Bukawa $\mathrm{H}$. Mandibular reconstruction using plates prebent to fit rapid prototyping 3-dimensional printing models ameliorates contour deformity. Head Face Med. 2014;10:45.

6. Rodby KA, Turin S, Jacobs RJ, Cruz JF, Hassid VJ, Kolokythas A, Antony AK. Advances in oncologic head and neck reconstruction: systematic review and future considerations of virtual surgical planning and computer aided design/computer aided modeling. J Plast Reconstr Aesthet Surg. 2014;67(9):1171-85.

7. Coleman SC, Burkey BB, Day TA, Resser JR, Netterville JL, Dauer $E$, Sutinis $E$. Increasing use of the scapula osteocutaneous free flap. Laryngoscope. 2000;110(9):1419-24.

8. Hidalgo DA, Pusic AL. Free-flap mandibular reconstruction: a 10-year follow-up study. Plast Reconstr Surg. 2002;110(2):438-49

9. Naros A, Weise H, Tilsen F, Hoefert S, Naros G, Krimmel M, Reinert $\mathrm{S}$, Polligkeit J. Three-dimensional accuracy of mandibular reconstruction by patient-specific pre-bent reconstruction plates using an "in-house" 3D-printer. J Craniomaxillofac Surg. 2018; 46(9):1645-1651.
Many authors underlined that the use of physical models of the mandible significantly decreases operation time and allows for obtainment of better aesthetic effect and shape of the lower face $(9,15,18)$. In our study, the aesthetic and functional outcomes were favorable by integrating $3 \mathrm{D}$ printing in the preoperative planning. The predictability of the results was also increased by a more exact modelling of the plates outside the operating room. Additionally, we considered that the use of surgical guides performed using the printed patientspecific model, also increased the precision of the plate positioning and contributed to the positive outcomes.

\section{CONCLUSIONS}

In our experience, integrating $3 \mathrm{D}$ printing in the preoperative planning of mandibular reconstruction procedures significantly improves the predictability and outcomes regarding the appearance and functional restoration. In addition, surgery time is decreased, while the confidence of the surgeons increases.

Conflict of interest: none declared Financial support: none declared

10. Liang Y, Jiang C, Wu L, Wang W, Liu Y, Jian X. Application of Combined Osteotomy and Reconstruction Pre-Bent Plate Position (CORPPP) Technology to Assist in the Precise Reconstruction of Segmental Mandibular Defects. J Oral Maxillofac Surg. 2017; 75(9):2026.e1-2026.e10.

11. Deek NF, Wei FC. Computer-Assisted Surgery for Segmental Mandibular Reconstruction with the Osteoseptocutaneous Fibula Flap: Can We Instigate Ideological and Technological Reforms? Plast Reconstr Surg. 2016;137(3):963-70.

12. Wilde F, Hanken H, Probst F, Schramm A, Heiland M, Cornelius CP. Multicenter study on the use of patient-specific CAD/CAM reconstruction plates for mandibular reconstruction. Int $\mathrm{J}$ Comput Assist Radiol Surg. 2015;10(12):2035-51.

13. Torroni A, Marianetti TM, Romandini M, Gasparini G, Cervelli D, Pelo S. Mandibular reconstruction with different techniques. J. Craniofac. Surg. 2015; 26(3):885-890.

14. Prisman E, Haerle SK, Irish JC, Daly M, Miles B, Chan H. Value of preoperative mandibular plating in reconstruction of the mandible. Head Neck. 2014;36(6):828-33.

15. Liu YF, Xu LW, Zhu HY, Liu SS. Technical procedures for templateguided surgery for mandibular reconstruction based on digital design and manufacturing. Biomed Eng Online. 2014;13:63.

16. Dupret-Bories A, Vergez S, Meresse T, Brouillet F, Bertrand G. Contribution of 3D printing to mandibular reconstruction after cancer. Eur Ann Otorhinolaryngol Head Neck Dis. 2018;135(2):133-136.

17. Liu PH, Wong TY V, Fang JJ, Chen KC, Hsiao JR, Huang JS. Stereolithographic Modeling Technique for Hemimandibular Reconstruction Report of a Case with Innovation Technique. Open Journal of Dentistry and Oral Medicine 2014; 2(1): 9-13.

18. Jacek B, Maciej P, Tomasz P, Agata B, Wiesław K, Radosław W, Filip G. 3D printed models in mandibular reconstruction with bony free flaps. J Mater Sci Mater Med. 2018;29(3):23. 